\title{
Diagnóstico de Progressão de Doença Coronária em Pacientes Previamente Tratados com Stents pela Tomografia Computadorizada de Múltiplos Detectores
}

\author{
Roberto Cândia', Afonso Akio Shiozaki', Mariana Fonte', Walther Ishikawa², Luiz Alberto Mattos' \\ Luiz Fernando Leite Tanajura', Fausto Feres ${ }^{1}$, Ibraim Masciarelli Pinto ${ }^{1}$, J. Eduardo Sousa', \\ Amanda G. M. R. Sousa ${ }^{1}$
}

\section{RESUMO}

Introdução: Pacientes previamente submetidos a intervenção coronária percutânea podem apresentar progressão de doença aterosclerótica ao longo do tempo, com o desenvolvimento de novas lesões em outros locais da árvore arterial coronária, o que pode representar um desafio diagnóstico. O objetivo deste trabalho foi avaliar a eficácia da tomografia computadorizada por múltiplos detectores na detecção da progressão de doença arterial coronária. Método: Entre julho de 2007 e julho de 2008, foram avaliados 60 pacientes previamente submetidos a implante de stents coronários e que apresentassem quadro clínico suspeito de recorrência de isquemia miocárdica tardia. Os pacientes foram divididos em dois grupos: grupo I (Gl), 30 pacientes avaliados pela tomografia computadorizada por múltiplos detectores; e grupo II (GII), 30 casos avaliados com cintilografia miocárdica. Todos os pacientes foram posteriormente submetidos a cinecoronariografia. Resultados: Os dados demográficos, a artéria tratada e o número de stents por paciente não diferiam entre os grupos. A tomografia computadorizada por múltiplos detectores identificou progressão de doença em $9(30 \%)$ pacientes e reestenose em $2(6,7 \%)$; a cintilografia miocárdica identificou $8(26,7 \%)$ casos de progressão de doença e $3(10 \%)$ de reestenose. A cinecoronariografia identificou 10 casos de progressão de doença no Gl (3 com estenoses entre $55 \%$ e $60 \%$ ) e 12 no Gll (4 com obstruções entre $50 \%$ e $60 \%$ ) e confirmou todas as suspeitas de reestenose. Conclusão: A tomografia computadorizada por múltiplos detectores demonstrou, em nossa casuística, bom desempenho na identificação da progressão de doença aterosclerótica em pacientes previamente

\section{ABSTRACT}

Diagnosis of the Progression of Coronary Artery Disease in Patients Previously Treated by Stents Using Multislice Computed Tomography

Background: Patients undergoing percutaneous coronary intervention may present progression of coronary artery disease in the late follow-up, leading to the development of new lesions in different sites of the coronary arteries, which may represent a diagnostic challenge. The goal of this paper was to evaluate the contribution of multislice computed tomography in this setting. Method: From July 2007 to July 2008 we analyzed 60 patients with suspect late myocardial ischemia after coronary stent implantation. Patients were divided in two groups. In Group I, 30 patients were evaluated by multislice computed tomography and in Group II, 30 patients were evaluated by myocardial scintigraphy. All patients were subsequently submitted to coronary angiography. Results: Demographic data, the treated vessel and the mean number of stents per patient were not significantly different in both groups. Multislice computed tomography identified progression of coronary disease in 9 patients (30\%) and restenosis in $2(6.7 \%)$; myocardial scintigraphy identified 8 cases $(26.7 \%)$ of disease progression and 3 cases $(10 \%)$ of restenosis. Coronary angiography revealed 10 cases of disease progression (3 cases with obstructions between 55\% and 60\%) in Group I and 12 in Group II (4 patients with obstructions between $50 \%$ and $60 \%$ ) and confirmed all suspected restenosis. Conclusion: Multislice computed tomography proved to be sensitive to detect progression of coronary artery disease in patients previously treated with stent implantation. Should

\footnotetext{
1 Instituto Dante Pazzanese de Cardiologia - São Paulo, SP, Brasil.

2 Hospital do Coração da Associação do Sanatório Sírio - São Paulo, SP, Brasil.

Correspondência: Ibraim Masciarelli Pinto. Instituto Dante Pazzanese de Cardiologia. Av. Dr. Dante Pazzanese, 500 - Ibirapuera - São Paulo, SP, Brasil - CEP 04012-180

E-mail: ipinto@cardiol.br

Recebido em: 2/6/2009 • 10/9/2009
} 
tratados com stents. Caso trabalhos adicionais, com maior número de pacientes, confirmem estes achados, a tomografia computadorizada por múltiplos detectores poderá incorporar mais essa indicação a seu espectro diagnóstico.

DESCRITORES: Tomografia computadorizada espiral. Doença da artéria coronariana/patologia. Stents. Cintilografia.

A intervenção percutânea com o implante de stents coronários é uma opção eficaz para o tratamento de obstruções no leito arterial coronário, e essa forma de tratamento representa hoje até dois terços dos procedimentos de revascularização em centros especializados. O advento das endopróteses revestidas com fármacos promoveu acentuada diminuição da reestenose, mas esses pacientes podem evoluir com progressão de doença coronária em locais diferentes dos previamente tratados, em especial após o primeiro ano de evolução ${ }^{1,2}$.

A progressão de doença coronária pode tornar-se um desafio para avaliação clínica em decorrência de uma série de fatores. Pacientes podem negligenciar os sintomas associados ao desenvolvimento de lesões coronárias, e o desempenho de testes de perfusão pode ficar limitado em decorrência de sequela de eventos isquêmicos prévios ou de desenvolvimento de lesões em segmentos miocárdicos contíguos ${ }^{3-5}$. O impacto clínico de lesões não diagnosticadas pode ser grave, especialmente aquelas localizadas em vasos que supram grandes áreas miocárdicas.

A tomografia computadorizada de múltiplos detectores tem tido sucesso na avaliação não-invasiva das artérias coronárias, mas seu papel no controle de pacientes tratados com stents ainda não é bem definido. Os resultados desse exame na avaliação de reestenose são satisfatórios quando as endopróteses têm diâmetro superior a $3 \mathrm{~mm}$ e se localizam em segmentos retos e relativamente proximais, havendo controvérsia na literatura quanto aos resultados obtidos quando tais condições ideais não são encontradas. Um dos principais obstáculos na avaliação da reestenose intrastent é o artefato causado pelo metal das próteses ${ }^{3,6}$.

Por outro lado, o estudo de progressão de doença coronária não sofre essas limitações. Como não há metal no local de interesse, o desempenho da tomografia pode ser semelhante àquele observado na população não previamente tratada com intervenção percutânea. Contudo, esse aspecto ainda não foi avaliado especificamente.

O objetivo deste trabalho foi avaliar o desempenho da tomografia computadorizada de múltiplos detectores na detecção da progressão de doença aterosclerótica em pacientes previamente tratados com stents coronários. larger scale studies confirm such findings this method could have an important role in the clinical evaluation of these patients.

DESCRIPTORS: Tomography, spiral computed. Coronary artery disease/pathology. Stents. Radionuclide imaging.

\section{MÉTODO}

Selecionamos 60 pacientes que haviam sido submetidos a implante de endopróteses e que apresentassem quadro clínico suspeito de recorrência de isquemia miocárdica tardia. Os critérios de inclusão compreendiam implante prévio de stent coronário, com suspeita clínica de isquemia miocárdica que houvesse surgido pelo menos seis meses após a intervenção. Foram excluídos pacientes com síndrome coronária aguda ou que tivessem recebido endopróteses para tratar reestenose intrastent, cirurgia de revascularização miocárdica prévia, creatinina $>2 \mathrm{mg} / \mathrm{dl}$ ou alergia a iodo.

Os pacientes foram divididos em dois grupos. No grupo I, foram incluídos 30 pacientes, avaliados por tomografia computadorizada de múltiplos detectores. No grupo II, foram incluídos 30 pacientes, avaliados por cintilografia miocárdica.

A tomografia compreendia a aquisição da fase arterial, feita com injeção de 1,2 ml/kg de contraste, na velocidade de $5 \mathrm{ml} / \mathrm{s}$, em equipamento de 64 detectores, sendo feito o preparo com nitrato sublingual e betabloqueador endovenoso, sempre que a frequência cardíaca estivesse acima de 65 batimentos por minuto, a menos que houvesse contraindicação para o uso desses medicamentos. Após o registro das imagens, a análise dos resultados era realizada em estação de trabalho equipada com programas específicos para a avaliação das artérias coronárias. A emissão de raios $\mathrm{X}$ era limitada a $100 \mathrm{kV}$, sempre que o peso corpóreo fosse inferior a $95 \mathrm{~kg}$, e a obtenção de imagens era acoplada ao eletrocardiograma, com emissão máxima de radiação durante a diástole e mínima durante a sístole.

A cintilografia foi realizada com injeção de sestamibi ligado ao tecnécio 99, compreendendo a fase de estresse provocado pelo esforço físico, seguida da obtenção de imagens em repouso. Não foram realizados exames com estímulo farmacológico.

Na tomografia procurou-se identificar a presença de obstruções intrastent ou em outros vasos coronários (Figura 1), enquanto na cintilografia procurou-se identificar defeitos transitórios ou permanentes da perfusão miocárdica, que sugerissem a presença de obstruções hemodinamicamente significativas. 

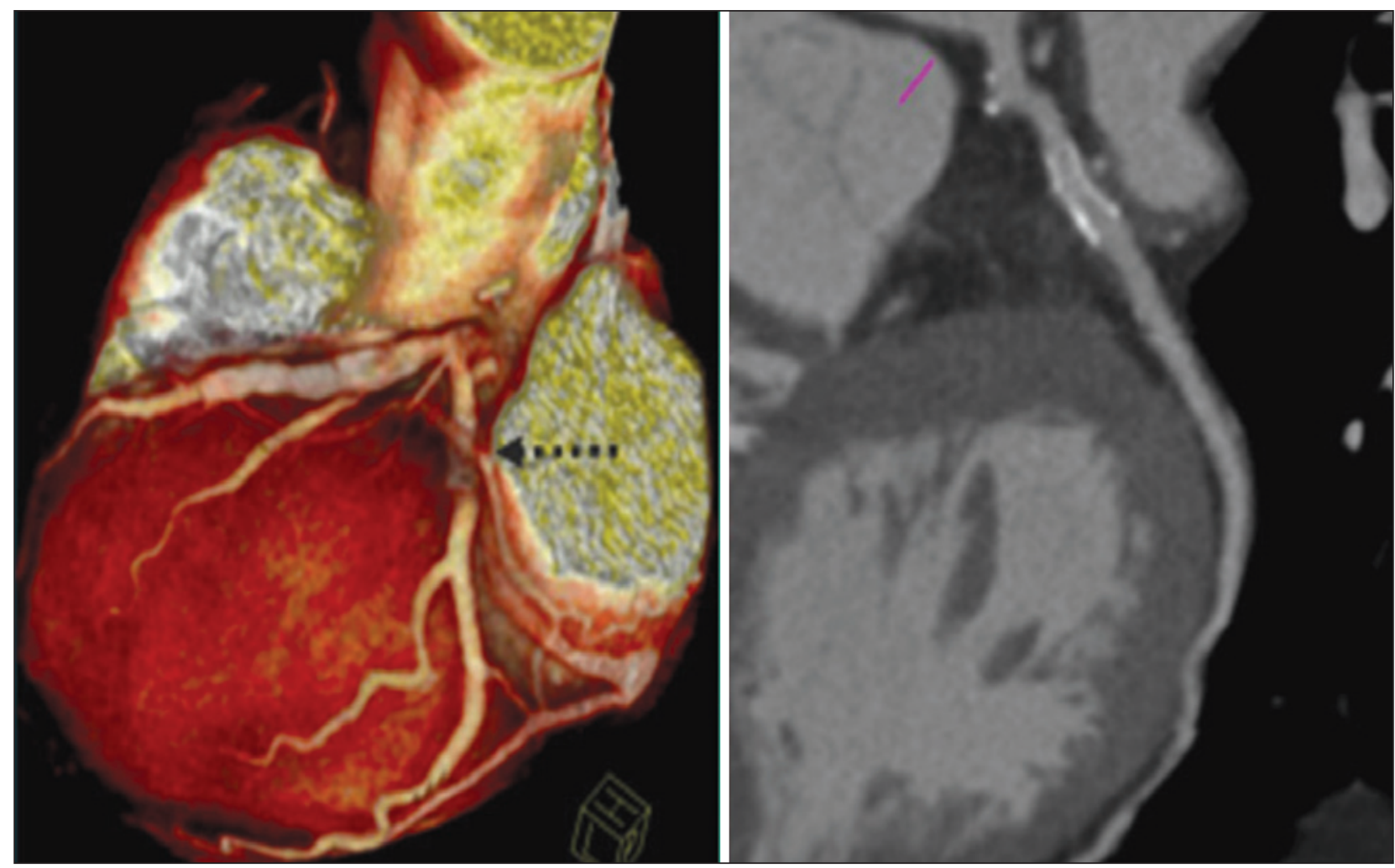

Figura 1 - A análise da angiotomografia é iniciada pela observação das reconstruções tridimensionais, que objetivam identificar a localização dos stents (seta). Em seguida, procede-se à avaliação da presença de obstruções intrastent ou em outros locais, a partir das reconstruções longitudinais.

\section{RESULTADOS}

As características demográficas, que foram semeIhantes nos dois grupos, encontram-se na Tabela 1.

O tempo médio de implante de stents era de $26 \pm$ 16 meses, sendo de $25 \pm 14$ meses no grupo I e de $28 \pm 17$ meses no grupo II.

Os vasos tratados encontram-se na Figura 2 e não diferiam nos dois grupos.

A cintilografia foi realizada sem complicações em todos os pacientes, com duração média de 3,6 \pm 1,2 horas e com dose média de radiação de $7,3 \pm$ 2,8 mSv. A tomografia computadorizada de múltiplos detectores também foi realizada sem complicações em todos os pacientes, com dose média de contraste de $123 \pm 48 \mathrm{ml}$ e duração média de $12 \pm 7$ minutos. A dose de radiação média foi de 5,6 \pm 3,5 mSv.

No grupo I (Figuras 3 e 4), de acordo com a tomografia computadorizada de múltiplos detectores, havia diminuição da luz maior que 50\% em vasos nãotratados em nove casos, dos quais três mostravam lesões entre $51 \%$ e $60 \%$ (uma artéria descendente anterior, um ramo diagonal e um ramo marginal), cinco exibiam estenoses entre $61 \%$ e $90 \%$ (dois ramos diagonais, uma artéria circunflexa e duas artérias coronárias direitas), e um apresentava oclusão do ramo diagonal. Em dois casos, a tomografia computadorizada de múltiplos detectores sugeriu presença de reestenose.

No grupo II, a cintilografia sugeriu presença de isquemia em território não-tratado em oito casos (parede anterior em dois, lateral em dois e inferior em quatro) e exibia imagens indicativas da recorrência de isquemia na parede irrigada por vaso tratado previamente em três casos, sugerindo reestenose.

A cinecoronariografia no grupo I demonstrou progressão de doença nos nove casos identificados pela tomografia computadorizada de múltiplos detectores e ainda identificou presença de estenose maior que $50 \%$ em vaso não-tratado (ramo marginal) em um caso, no qual a tomografia havia indicado presença de estenose entre $40 \%$ e $50 \%$.

No grupo II, o exame invasivo confirmou a suspeita de progressão de doença coronária nos oito casos identificados pela cintilografia, mas também apontou presença de obstruções acima de 50\% em dois outros casos (um ramo diagonal com obstrução de $60 \%$ e um ramo descendente posterior com obstrução de 55\%).

Houve confirmação de presença de reestenose em todos os casos sugeridos pelos exames nãoinvasivos. 
TABELA 1

Características demográficas

\begin{tabular}{|c|c|c|}
\hline & Grupo I & Grupo II \\
\hline Idade, anos (média \pm DP) & $57 \pm 12$ & $53 \pm 14$ \\
\hline Sexo masculino, $\mathrm{n}$ & 26 & 22 \\
\hline Hipertensão, n & 21 & 19 \\
\hline Diabetes melito, $\mathrm{n}$ & 12 & 15 \\
\hline $\begin{array}{l}\text { Nível elevado de LDL, } \\
\text { mg/dl (média } \pm \text { DP) }\end{array}$ & $132 \pm 36$ & $143 \pm 29$ \\
\hline
\end{tabular}

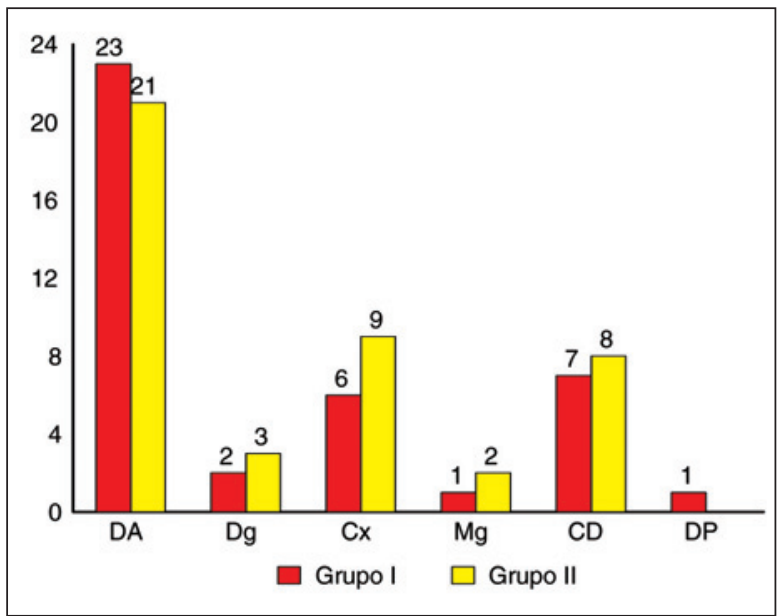

Figura 2 - Vaso tratado originalmente nos pacientes de ambos os grupos. $\mathrm{CD}=$ artéria coronária direita; $\mathrm{Cx}=$ artéria circunflexa; $\mathrm{DA}=$ artéria descendente anterior; $\mathrm{Dg}=$ ramo diagonal; $\mathrm{DP}=$ artéria descendente posterior; $\mathrm{Mg}=$ ramo marginal

\section{DISCUSSÃO}

A pesquisa clínica de progressão de doença coronária é importante, e ainda representa um desafio na prática clínica, exigindo grande emprego de exames e recursos para esse fim. A relevância do tema tem como base o fato de que pacientes com doença coronária detectada previamente são subgrupo com maior propensão ao desenvolvimento de novas placas ateroscleróticas em outros sítios da árvore coronária ${ }^{3}$.

As dificuldades que o diagnóstico apresenta são decorrentes de múltiplas causas. Muitas vezes, os pacientes, por acreditarem ter sido tratados definitivamente, podem não valorizar os sintomas que revelariam a presença de novas obstruções nas artérias coronárias. Em outras situações, a lesão recém-desenvolvida pode estar presente em vasos que irrigam áreas contíguas àquelas tratadas originariamente, o que pode dificultar a interpretação de exames detectores de

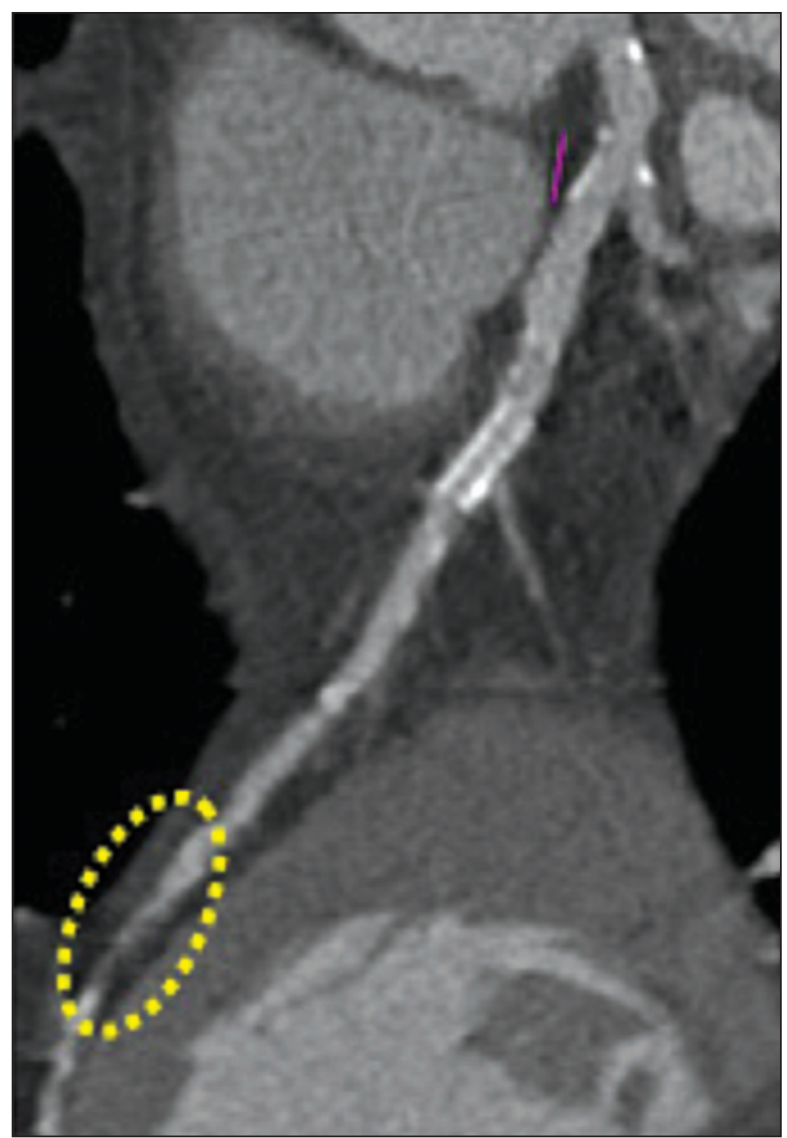

Figura 3 - Exame de angiotomografia revelando progressão de doença coronária no mesmo vaso em que, 30 meses antes, houve implante de stent (artéria descendente anterior) e que não mostrava sinais de reestenose.

isquemia. Tais dificuldades também podem ser decorrentes da presença de eventos isquêmicos prévios em áreas vizinhas às regiões nas quais se desenvolveu nova lesão. Assim sendo, a possibilidade de se dispor de um método de avaliação anatômica para a investigação não-invasiva de progressão de doença coronária é atraente ${ }^{3}$. Tal uso clínico parece ter potencial para a aplicação prática, pois a tomografia computadorizada de múltiplos detectores, ao analisar pacientes na fase tardia após o implante de stents coronários, pode identificar a presença de obstruções em vasos não-tratados.

Ao contrário do relatado em estudos iniciais, a tomografia computadorizada de múltiplos detectores tem apresentado resultados satisfatórios na avaliação da detecção e do grau de estenose das artérias coronárias. A despeito dos bons resultados obtidos com o uso dessa tecnologia para avaliar lesões em artérias coronárias e enxertos de veia safena, o uso da tomografia computadorizada de múltiplos detectores para avaliar pacientes tratados com stents coronários ainda é controverso ${ }^{7,8}$. 
Cândia R, et al. Diagnóstico de Progressão de Doença Coronária em Pacientes Previamente Tratados com Stents pela Tomografia Computadorizada de Múltiplos Detectores. Rev Bras Cardiol Invas. 2009;17(3):352-7.
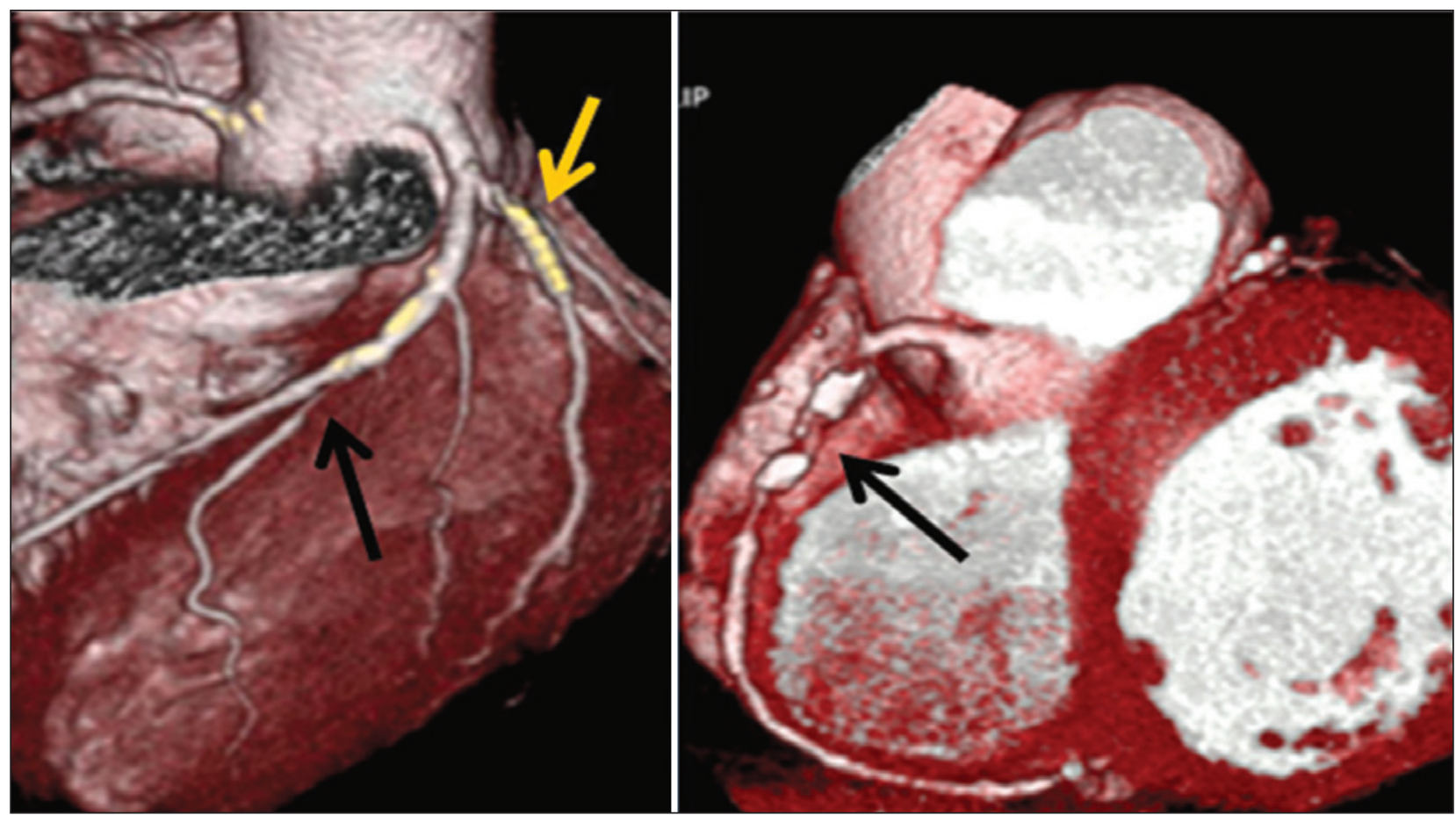

Figura 4 - Angiotomografia de paciente que havia recebido implante de stent em ramo marginal (seta amarela), no qual houve progressão de doença arterial coronária no segundo ramo diagonal e na artéria coronária direita (setas pretas).

Os stents ainda trazem dificuldade à interpretação das imagens, em grande parte em decorrência de seu arcabouço metálico. Na medida em que a radiação incide sobre os stents, as hastes absorvem os raios de mais baixa energia e permitem a passagem dos raios de alta energia, levando à formação de brilho exagerado de sua estrutura. Esse artefato, mais conhecido como blooming artifact, pode dificultar a avaliação das regiões peristent, e varia na dependência das dimensões da prótese, de sua arquitetura e da condição da artéria no local tratado 5,9 . Também há variação da qualidade de imagem de equipamento para equipamento, o que faz com que os resultados da literatura sejam conflitantes. Devemos destacar progressos que tornaram as imagens da tomografia computadorizada de múltiplos detectores mais precisas, como os novos protocolos de imagem que reduziram a dose de radiação, tornando esse exame ainda mais seguro para os pacientes ${ }^{10}$. A dose de radiação que empregamos foi inferior àquela descrita habitualmente em trabalhos que avaliam a tomografia computadorizada de múltiplos detectores na análise das artérias coronárias, alcançada com a redução da frequência cardíaca, o que faz com que a modulação da dose de radiação seja mais efetiva. Como essa dose máxima é aplicada ao final de diástole e a dose ao final de sístole é muito menor, o controle dos batimentos cardíacos faz com que o período no qual se empregam níveis mais altos de energia seja reduzido. Além disso, sempre que os pacientes apresentavam peso corpóreo inferior a $95 \mathrm{~kg}$, a voltagem do tubo era fixada em 100 kV, ao contrário dos $120 \mathrm{kV}$ empregados tradicionalmente. Isso teve grande eficácia em reduzir a dose de radiação, mas não diminuiu a qualidade das imagens a ponto de impedir sua análise adequada.

Essa impressão é confirmada não apenas porque os investigadores lograram avaliar todos os segmentos coronários na totalidade dos pacientes, mas também porque a tomografia computadorizada de múltiplos detectores teve sucesso na identificação das obstruções presentes nas artérias coronárias. No grupo avaliado apenas pela tomografia computadorizada de múltiplos detectores, nos casos em que houve discordância entre o exame não-invasivo e a angiografia convencional, as obstruções apresentavam diminuição moderada da luz e o grau de diferença entre os exames não foi superior a $10 \%$. O desempenho da cintilografia, em nossa amostra, também foi satisfatório, e, mais uma vez, quando houve falta de concordância entre esse exame e a angiografia, tratava-se de obstruções moderadas.

Embora não tenha existido comparação direta entre os grupos e esse não seja o objetivo deste trabalho, poder-se-ia considerar que os resultados da tomografia não foram superiores aos da cintilografia, o que representaria uma dificuldade para a implementação prática desse exame. Contudo, o tempo de permanência hospitalar e a facilidade de realização podem ser argumentos favoráveis à utilização da 
tomografia computadorizada de múltiplos detectores na prática. Por outro lado, também é válido o argumento de que a tomografia computadorizada de múltiplos detectores não apresentou resultado inferior. Assim, essa dúvida justifica o planejamento e a realização de um outro estudo com a finalidade específica de comparar o desempenho de ambos os métodos nesse cenário.

Deve ainda ser destacado que a tomografia computadorizada de múltiplos detectores não teve seus resultados limitados pelas dimensões das artérias tratadas, mostrando bons resultados tanto no caso das artérias coronárias principais como no caso de ramos secundários.

Raros casos de reestenose que se manifestaram clinicamente foram observados em nosso trabalho, os quais foram adequadamente visualizados pelos métodos não-invasivos. Contudo, esse item não fazia parte do planejamento inicial de nosso trabalho e esse achado não deve ser valorizado, sendo mais uma vez necessários outros estudos para avaliar esse objetivo.

Naturalmente existem limitações a nosso estudo. Trata-se de trabalho feito em dois serviços, mas com a mesma equipe médica, podendo representar muito mais a experiência de um serviço que o desempenho da metodologia. Além disso, nossa amostra é algo limitada, mas esses resultados encorajaram a realização de um estudo em mais ampla escala, que poderá trazer resultados ainda mais solidificados.

\section{CONCLUSÃO}

A tomografia computadorizada de múltiplos detectores demonstrou, em nossa casuística, bom desempenho no monitoramento da progressão de obstruções coronárias em pacientes previamente tratados com stents. Caso trabalhos adicionais, com maior número de pacientes, confirmem estes achados, a tomografia computadorizada de múltiplos detectores poderá incorporar mais essa indicação a seu espectro diagnóstico.

\section{CONFLITO DE INTERESSES}

Os autores declararam inexistência de conflito de interesses.

\section{REFERÊNCIAS BIBLIOGRÁFICAS}

1. Park MW, Seung KB, Kim PJ, Park HJ, Yoon SG, Baek JY, et al. Long-term percutaneous coronary intervention rates and associated independent predictors for progression of nonintervened nonculprit coronary lesions. Am J Cardiol. 2009; 104(5):648-52.

2. Sousa JE, Costa MA, Abizaid A, Abizaid AS, Feres F, Pinto $I M$, et al. Lack of neointimal proliferation after implantation of sirolimus-coated stents in human coronary arteries: a quantitative coronary angiography and three-dimensional intravascular ultrasound study. Circulation. 2001;103(2):192-5.

3. Pinto IM, Sousa AG, Ishikama W, Mattos LA, Abizaid A, Feres $\mathrm{F}$, et al. Late outcome of sirolimus-eluting stents: comparison of multidetector computed tomography with quantitative coronary angiography and intravascular ultrasound. Arq Bras Cardiol. 2006;87(5):575-82.

4. Cademartiri F, Schuijf JD, Pugliese F, Mollet NR, Jukema JW, Maffei E, et al. Usefulness of 64-slice multislice computed tomography coronary angiography to assess in-stent restenosis. J Am Coll Cardiol. 2007;49(22):2204-10.

5. Halon DA, Gaspar T, Adawi S, Peled N, Lewis BS. Coronary stent assessment on multidetector computed tomography: source and predictors of image distortion. Int J Cardiol. 2008;128(1):62-8.

6. Gopalakrishnan P, Wilson GT, Tak T. Accuracy of multislice computed tomography coronary angiography: a pooled estimate. Cardiol Rev. 2008;16(4):189-96.

7. Nieman K, Oudkerk M, Rensing BJ, van Ooijen P, Munne A, van Geuns RJ, et al. Coronary angiography with multi-slice computed tomography. Lancet. 2001;357(9256):599-603.

8. Kruger S, Mahnken AH, Sinha AM, Borghans A, Dedden K, Hoffmann R, et al. Multislice spiral computed tomography for the detection of coronary stent restenosis and patency. Int J Cardiol. 2003;89(2-3):167-72.

9. Mahnken AH, Buecker A, Wildberger JE, Ruebben A, Stanzel $S$, Vogt F, et al. Coronary artery stents in multislice computed tomography: in vitro artifact evaluation. Invest Radiol. 2004; 39(1):27-33.

10. Gutstein A, Dey D, Cheng V, Wolak A, Gransar H, Suzuki $Y$, et al. Algorithm for radiation dose reduction with helical dual source coronary computed tomography angiography in clinical practice. J Cardiovasc Comput Tomogr. 2008; 2(5):311-22. 